\title{
Factores asociados \\ a la práctica reflexiva \\ en estudiantes de pedagogía
}

Factors Associated with the Reflective Practice in Pedagogy Students

Fecha de recepción: 30 DE NOVIEMBRE DE 2016 / Fecha de aceptación: 7 DE JUNIO DE 2018 / Fecha de disponibilidad en línea: ENERO DE 2019

Álvaro luis Salinas-Espinosa asalinase@uc.cl

Pontificia Universidad Católica de Chile, Chile https://orcid.org/0000-0001-9655-761X

Tamara Rozas-Assael t.rozas@ucl.ac.uk

Pontificia Universidad Católica de Chile, Chile https://orcid.org/0000-0002-0272-7831

Pablo Cisternas-Alarcón pacister@uc.cl

Pontificia Universidad Católica de Chile, Chile https://orcid.org/0000-0003-2557-4185

Carlos González-Ugalde cgonzalu@uc.cl

Pontificia Universidad Católica de Chile, Chile https://orcid.org/0000-0002-3154-0777

\begin{abstract}
Resumen
Este artículo describe la frecuencia con la cual estudiantes de pedagogía realizan prácticas reflexivas, y analiza factores que pueden explicar esta frecuencia. Para ello se empleó una perspectiva cuantitativa que incluyó una encuesta a una muestra de 650 estudiantes de 134 programas de formación de profesores en Chile. Los resultados muestran que los factores más importantes para explicar la práctica reflexiva se relacionan con el supervisor. Los factores relacionados con los compañeros de estudio y con la discusión sobre la profesión docente contribuyen menos a la práctica reflexiva.
\end{abstract}

\section{Palabras clave}

Estudiante de prácticas; formación de docentes; práctica pedagógica

\begin{abstract}
This article describes how frequently the pedagogy students carry out reflective practices. It also analyze the factors that can explain the frequency. To do so, a quantitative perspective was used including a survey applied to sample of 650 students from 134 teacher training programs in Chile. The results indicate that the most important factors to explain the reflective practice are related to the supervisor. The factors related to the classmates and to the discussion on the teacher career have a lower contribution to the reflective practice.
\end{abstract}

\section{Keywords}

Trainees; teacher education; teaching practice

Para citar este artículo / To cite this article

Salinas-Espinosa, Á. L.; Rozas-Assael, T.; Cisternas-Alarcón, P. \& González-Ugalde, C. (2019). Factores asociados a la práctica reflexiva en estudian-tes de pedagogía. magis, Revista Internacional de Investigación en Educación, 11 (23), 95-114. doi: 10.11144/Javeriana.m11-23.fapr 


\section{Introducción}

Durante las últimas décadas, la práctica reflexiva ha aparecido como un atributo deseable para los profesores en ejercicio y como un resultado valorado por numerosos programas de formación de profesores (Collin, Karsenti \& Komis, 2012). En América Latina, varios sistemas educativos han incorporado la reflexividad como un elemento importante del desempeño docente (Meckes, 2014). Este creciente interés en la práctica reflexiva ha generado copiosa investigación sobre qué es la práctica reflexiva, cómo puede ser desarrollada y cómo se relaciona con la enseñanza de los profesores y el aprendizaje de los estudiantes en las escuelas. Sin embargo, la evidencia no es concluyente aún respecto de si la práctica reflexiva puede desarrollarse durante la formación inicial de profesores, ni en cómo hacerlo ni tampoco si su desarrollo tiene algún efecto en la sala de clase (Beauchamp, 2014; Collin, Karsenti \& Komis, 2012). Tampoco hay mucha investigación que compare distintos factores relacionados con la formación y el desempeño docente, de modo que identifique aquellos que más contribuyan al desarrollo de la práctica reflexiva (Beauchamp, 2014; Collin, Karsenti \& Komis, 2012). Además, la investigación se ha realizado fundamentalmente en contexto anglosajón. Muy poco se ha publicado en América Latina sobre este tema.

Este artículo busca aportar al conocimiento sobre los factores que contribuyen de manera más significativa al desarrollo de la práctica reflexiva en estudiantes de pedagogía en Chile. Para este propósito, este artículo describe la frecuencia con la cual los estudiantes de pedagogía se involucran en prácticas reflexivas, y analiza algunos factores relacionados con esa frecuencia. Los factores considerados se refieren específicamente a la cantidad de prácticas profesionales que los estudiantes de pedagogía realizan a lo largo de su formación, a las actividades de discusión entre estudiantes de pedagogía y quienes supervisan esta experiencia de práctica, y a las actividades de observación y retroalimentación que reciben los estudiantes de pedagogía sobre su desempeño en la práctica profesional.

\section{Práctica reflexiva}

La literatura sobre la práctica reflexiva no ha logrado definir de manera unívoca este concepto (Beauchamp, 2014; Collin, Karsenti \& Komis, 2012). Sin embargo, en este trabajo se entiende práctica reflexiva como el examen, comprensión y análisis crítico del trabajo docente a partir de las distintas formas de conocimiento profesional, en particular, del conocimiento sobre la enseñanza, el aprendizaje y el contexto donde se realiza el trabajo docente, adquiridos durante la formación y durante la experiencia práctica del profesor (Beauchamp, 2014; Collin, Karsenti \& Komis, 2012; Moore-Russo \& Wilsey, 2014).

Esta reflexión docente implica muy centralmente lo que Donald A. Schön llama enmarcamiento y reenmarcamiento que los profesionales hacen de las situaciones complejas que enfrentan en su práctica, a partir de marcos interpretativos elaborados con el conocimiento del que disponen (Schön, 1983). Estos marcos interpretativos pueden poner en relieve distintos focos, a partir de los cuales se hacen el enmarcamiento y el reenmarcamiento. Estos focos pueden concentrarse sobre la acción del propio docente (su enseñanza), sobre cómo esa acción tiene unas ciertas consecuencias (en el aprendizaje de los alumnos) y sobre el marco más amplio donde se produce esta acción (las condiciones e implicancias del trabajo docente) (Beauchamp, 2014; Collin, Karsenti \& Komis, 2012; Moore-Russo \& Wilsey, 2014). 
Numerosos trabajos han analizado el desarrollo de la práctica reflexiva al identificar niveles crecientes en complejidad y alcance del análisis reflexivo (Sparks-Langer, Simmons, Pasch, Colton \& Starko, 1990). Barbara Larrivee (2008), por ejemplo, distingue tres niveles de reflexión y un cuarto llamado prerreflexivo. Este último se caracteriza por la reacción automática de los profesores a las situaciones en la sala de clases sin considerar otras alternativas de respuesta. En este nivel, las cosas se dan por sentadas sin cuestionamiento. El nivel de reflexión superficial pone atención en las estrategias y métodos para alcanzar unos determinados fines, sin preguntarse por el valor de esos fines. El nivel de reflexión pedagógica se caracteriza por que los profesores aplican conocimiento del campo profesional y creencias sobre qué es una práctica de calidad. Por último, en el nivel de reflexión crítica, los profesores abordan las implicancias morales y éticas y las consecuencias de sus prácticas en los estudiantes.

La jerarquía que suponen los niveles de reflexión descritos por la literatura ha generado debate porque supone de manera implícita que hay unos niveles más complejos y adecuados que otros para la labor docente, organizados linealmente (Collin, Karsenti \& Komis, 2012). Tomando distancia de esto, algunos autores plantean que eso que usualmente se ha descrito como niveles corresponde más bien a tipos de reflexividad, los que pueden ser más o menos relevantes según los distintos contextos y situaciones en que se desarrolla el trabajo (Collin, Karsenti \& Komis, 2012; Leijen, Valtna, Leijen \& Pedaste, 2012). Esto significa que los distintos tipos de reflexividad contribuyen a comprender y mejorar la práctica docente en el contexto de la sala de clases. Sin embargo, la investigación tiende a mostrar que los estudiantes de pedagogía más bien describen su propia enseñanza con escaso uso de distinciones propias del campo pedagógico y no consideran las consecuencias de su enseñanza en el aprendizaje de los alumnos ni las mediaciones sociales y políticas de su trabajo (Collin, Karsenti \& Komis, 2012; Mansvelder-Longayroux, Beijaard \& Verloop, 2007). Una primera pregunta de investigación abordada en este trabajo es: entre los estudiantes de último año de formación pedagogía en Chile, ¿se observa también una práctica reflexiva que considera poco el aprendizaje de los alumnos y el contexto en el que se desarrolla la práctica docente?

\section{La práctica reflexiva en la formación inicial de profesores}

Buena parte de la investigación sobre la práctica reflexiva en educación se ha concentrado en estudiar su desarrollo durante la formación inicial de los docentes (Collin, Karsenti \& Komis, 2012).

Esta investigación ha mostrado que uno de los espacios más importantes para el desarrollo de la reflexividad docente es la práctica profesional que los estudiantes de pedagogía deben llevar a cabo a lo largo de su formación (Collin, Karsenti \& Komis, 2012; Jones \& Ryan, 2014). La razón de ello es lo fundamental que es para el desarrollo de la reflexividad el vínculo con la práctica en contextos reales que ofrecen estas actividades curriculares (Collin, Karsenti \& Komis, 2012; Gelfuso \& Dennis, 2014). La cantidad de prácticas profesionales que un estudiante de pedagogía tiene a lo largo de su formación puede ofrecer más o menos oportunidades para enriquecer su aprendizaje con la experiencia directa en contextos de desempeño (Darling-Hammond \& Lieberman, 2012).

Sin embargo, el cómo se utilicen esas oportunidades es fundamental para el desarrollo de la práctica reflexiva (Jones \& Ryan, 2014). La investigación se ha concentrado en analizar cómo las actividades de supervisión y el supervisor de la práctica profesional inciden en el desarrollo de la reflexión de los futuros docentes (Bates, Ramírez \& Drits, 2009). La supervisión es 
entendida como una actividad curricular que ocurre a lo largo de la formación docente, en la cual se ofrecen oportunidades para que los estudiantes tengan una experiencia directa en una sala de clases y para que esa experiencia sea discutida con compañeros de práctica profesional y con un supervisor (Solís, Núñez, Contreras, Rittershaussen, Montecinos \& Walker, 2011). El supervisor es usualmente un profesional que depende de la institución formadora y acompaña a los estudiantes de pedagogía durante su experiencia de práctica. Para ello, observa su desempeño en la sala de clases y se reúne periódicamente con ellos para analizar esta experiencia (Solís, Núñez, Contreras, Rittershaussen, Montecinos \& Walker, 2011).

La investigación ha analizado cómo los temas sobre los cuales se concentra la discusión durante la supervisión (sobre qué se discute) y su estructura (cómo se discute) inciden en las prácticas reflexivas. También se han dedicado esfuerzos a analizar la observación que hace el supervisor del desempeño de los estudiantes, usando distintos recursos para registrar la experiencia, y a estudiar cómo estos recursos ofrecen oportunidades para la discusión y retroalimentación sobre la experiencia.

En relación con los temas, la discusión con el supervisor sobre la enseñanza y el aprendizaje en la sala de clases puede estimular la reflexión de los estudiantes de pedagogía (Collin, Karsenti \& Komis, 2012; Jones \& Ryan, 2014), así como la discusión sobre la profesión docente y las condiciones en las que se desenvuelve (Bates, Ramírez \& Drits, 2009; Sparks-Langer, Simmons, Pasch, Colton \& Starko, 1990).

En relación con la estructura de la discusión, la investigación no es concluyente. Por ejemplo, el trabajo de Elizabeth Jaeger (2013) muestra que el tipo de discusión entre los estudiantes de pedagogía y el supervisor es importante. Cuando las discusiones son más directivas, más instructivas, los estudiantes de pedagogía pueden movilizar sus creencias y saber, conectar la teoría con la práctica y analizar los contextos en los cuales desarrollan su labor (Jaeger, 2013). Cuando la discusión es menos directiva, menos instructiva, los estudiantes tienen espacio para construir sus propias representaciones de la enseñanza y para explorar nuevas vías de reflexión (Moore-Russo \& Wilsey, 2014). Sin embargo, otros trabajos han encontrado que la estructura de la discusión realizada durante la supervisión con la ayuda de herramientas digitales no es un factor importante para desarrollar una reflexión profunda (Jones \& Ryan, 2014).

La retroalimentación que el supervisor y los otros estudiantes de pedagogía hagan durante la práctica y la discusión sobre el desempeño de los practicantes en la sala de clases son factores clave para promover el desarrollo de la práctica reflexiva (Gelfuso \& Dennis,
2014; Harford \& MacRuairc, 2008). La investigación muestra también que observar prácticas de enseñanza de otros estudiantes de pedagogía contribuye a desarrollar la práctica reflexiva (Harford \& MacRuairc, 2008), y que el uso de videos junto con formas específicas de interacción con "otros conocedores" puede contribuir al desarrollo de altos niveles de reflexión durante la experiencia de práctica profesional (Gelfuso \& Dennis, 2014).

Como se puede apreciar, muchas investigaciones han analizado mecanismos específicos que pueden contribuir al desarrollo de la práctica reflexiva. Sin embargo, pocos trabajos han estudiado de manera comparativa cuáles contribuyen más al desarrollo de la práctica reflexiva.

De esto surge la segunda pregunta de investigación que se aborda en este texto: ¿cuáles son las actividades específicas desarrolladas en el contexto de la práctica profesional que contribuyen más al desarrollo de la práctica reflexiva en estudiantes de pedagogía que están finalizando sus estudios? Para responder a esta pregunta, los estudiantes de pedagogía fueron consultados sobre la cantidad de prácticas profesionales en las cuales ellos han participado durante su formación y sobre la frecuencia de actividades específicas desarrolladas en el contexto de la supervisión. Las actividades consideradas en este estudio son aquellas que han sido identificadas por la investigación precedente como importantes para promover la práctica reflexiva. Se ha descartado el estudio de la estructura de la discusión por la falta de claridad en la investigación sobre su contribución a la práctica reflexiva y por la dificultad que implica recoger información de esta naturaleza en una investigación de carácter cuantitativo como la que se presenta aquí.

\section{Metodología}

Este estudio es cuantitativo. Describe la frecuencia con la cual los estudiantes de pedagogía reflexionan sobre focos específicos de su experiencia de práctica profesional, y analiza factores asociados a la práctica profesional y la supervisión que pueden ayudar a explicar esta frecuencia.

\section{Preguntas de investigación e hipótesis de estudio}

Como ya se ha dicho, la primera pregunta de investigación que guía este trabajo es:

Entre los estudiantes de último año de formación pedagogía en Chile, ¿se observa también una práctica reflexiva que considera poco el aprendizaje de los alumnos y el contexto en el que se desarrolla la práctica docente? 
Como se ha dicho, en distintos contextos nacionales la evidencia muestra que la reflexión de los estudiantes de pedagogía es limitada, y se concentra más bien en el examen de la propia enseñanza y menos en la reflexión sobre el aprendizaje o el contexto en el que se desarrolla la actividad docente (Collin, Karsenti \& Komis, 2012; Leijen, Valtna, Leijen \& Pedaste, 2012; Mansvelder-Longayroux, Beijaard \& Verloop, 2007). Para contribuir con evidencia cuantitativa obtenida de numerosos programas de formación, este estudio propone como hipótesis 1:

En Chile, los estudiantes de pedagogía en último año de formación reflexionarán con mayor frecuencia sobre la enseñanza y con menos frecuencia sobre el aprendizaje y el contexto en el cual se desarrolla su trabajo.

La segunda pregunta que guía este trabajo es:

¿Cuáles son las actividades específicas desarrolladas en el contexto de la práctica profesional que contribuyen más al desarrollo de la práctica reflexiva en estudiantes de pedagogía que están finalizando sus estudios en Chile?

La experiencia de práctica profesional debiera ser fundamental para el desarrollo de la práctica reflexiva en estos estudiantes, dado el acceso privilegiado que ofrece a los contextos de desempeño (Collin, Karsenti \& Komis, 2012; Gelfuso \& Dennis, 2014; Jones \& Ryan, 2014), de modo tal que la hipótesis 2 es:

Para los estudiantes aquí considerados, a mayor cantidad de prácticas profesionales, más frecuencia de práctica reflexiva.

Junto con la cantidad de prácticas que realicen los estudiantes, las oportunidades que ofrezca la supervisión para aprender de esta experiencia debiera ser importante para el desarrollo de la reflexividad (Gelfuso \& Dennis, 2014), de modo tal que la hipótesis 3 es:

Mientras más observación, retroalimentación y análisis sobre el desempeño de los estudiantes de pedagogía ofrezca el supervisor, mayor frecuencia de práctica reflexiva de los estudiantes de pedagogía.

Y la hipótesis 4 es:

la retroalimentación y el análisis del desempeño de los estudiantes que hace el supervisor son el factor que mayor peso tiene, en comparación con los otros considerados en este estudio, para explicar la frecuencia de práctica reflexiva de los estudiantes de pedagogía en Chile. Otros factores tales como la discusión con compañeros, la discusión sobre la profesión docente y la discusión sobre las relaciones en la escuela tendrán un peso menor que las oportunidades que ofrezca la supervisión.

Los compañeros están en la misma condición de practicantes y no disponen de distinciones que agreguen algo muy distinto a la reflexión que cada uno de ellos pueda hacer individualmente (no son "otros conocedores", siguiendo a Gelfuso \& Dennis, 2014). La discusión sobre la profesión docente y sobre las relaciones en la escuela puede abordar aspectos como las condiciones de trabajo y el rol de los profesores (Bates, Ramírez \& Drits, 2009; Sparks-Langer, Simmons, Pasch, Colton \& Starko, 1990), pero abordan menos aspectos como el aprendizaje y la enseñanza en la sala de clases. Estos argumentos sugieren la hipótesis 5 : 
La discusión con los compañeros tendrá una relación positiva y significativa estadísticamente, pero tendrá un peso menor en la explicación de la práctica reflexiva.

La hipótesis 6:

La discusión sobre la profesión docente tendrá una relación positiva y significativa estadísticamente, pero tendrá un peso menor en la explicación de la práctica reflexiva.

Y la hipótesis 7:

La discusión sobre las relaciones con colegas y padres tendrá una relación positiva y significativa estadísticamente, pero tendrá un peso menor en la explicación de la práctica reflexiva.

\section{Contexto: la formación inicial de profesores en Chile}

En 2013, 62 instituciones ofrecían formación inicial de profesores para los niveles preescolar, primario y secundario en el país, con una matrícula aproximada de 70.000 estudiantes.

La mayoría de los programas tiene una duración de 5 años, en los que los cursos sobre pedagogía son concurrentes con los cursos de formación en las disciplinas (Ingvarson, Schwille, Tatto, Rowley, Peck \& Senk, 2013). Menos programas tienen una duración de un año, y se concentran en formación pedagógica para estudiantes que han obtenido previamente un grado académico en alguna disciplina relacionada con el currículo escolar (historia, matemáticas, etc.).

Además de la formación pedagógica y disciplinar, los programas ofrecen a los estudiantes de pedagogía oportunidades para realizar experiencias en contextos reales de desempeño en escuelas (Ingvarson, SchwiIle, Tatto, Rowley, Peck \& Senk, 2013). Estas prácticas profesionales tienden a ser progresivas: a medida que avanzan en su formación, los estudiantes de pedagogía pasan más tiempo en las escuelas y asumen roles más centrales en la sala de clases (Solís, Núñez, Contreras, Rittershaussen, Montecinos \& Walker, 2011). Aunque la duración de la práctica profesional final es variable según los distintos programas de formación, la experiencia tiende a durar un semestre completo; en este tiempo, los estudiantes de pedagogía observan clases, apoyan al profesor a cargo del curso, realizan tareas menores y enseñan directamente algunas clases (Solís, Núñez, Contreras, Rittershaussen, Montecinos \& Walker, 2011).

\section{Variables}

La variable dependiente de este estudio es el reporte de la frecuencia de prácticas reflexivas desarrolladas por estudiantes de pedagogía durante su práctica profesional. Esta variable ha sido medida usando una versión modificada de la escala de reflexividad elaborada por Barbara Larrivee (2008).

La elección de esta escala para el presente estudio se justifica por varios elementos. En primer lugar, el enfoque usual en la investigación sobre prácticas reflexivas en educación considera enfoques cualitativos, generalmente expresados en el análisis de textos que los sujetos deben producir (Collin, Karsenti \& Komis, 2012; Jaeger, 2013). Las ventajas de este enfoque son numerosas e importantes. Sin embargo, uno de los costos de este predominio ha sido la escasez de estudios a gran escala con muestras grandes que permitan probar hipótesis, comparar grupos y describir cómo 
se distribuye la reflexión en una población mayor (Collin, Karsenti \& Komis, 2012; Jaeger, 2013). Para recoger información sobre una muestra grande, los instrumentos de autorreporte son una opción frecuente en investigación social (Podsakoff, MacKenzie \& Podsakoff, 2012) y han sido empleados en estudios sobre práctica reflexiva en el campo de la salud (Kember, Leung, Jones, Loke, McKay, Sinclair, Tse, Webb, Yuet Wong, Wong \& Yeung, 2000; Sobral, 2001). Además de la masividad, otra ventaja de este tipo de instrumento es su anonimato, lo que es especialmente importante en este estudio dado que se pedía a los estudiantes reportar sobre la frecuencia de prácticas reflexivas propias, pero también sobre la frecuencia de actividades realizadas durante la supervisión. En este contexto, el reporte de los supervisores resulta potencialmente sesgado. Con todo, para reducir el riesgo de sesgo por deseabilidad social en este estudio, se tomaron varias medidas: se recogió información anónima, los estudiantes debían firmar un consentimiento que explicaba el uso de la información y reafirmaba el anonimato de la información y la encuesta era autoaplicada, de manera que quien responde no está en una situación interpersonal en que ponga en juego su imagen (Podsakoff, MacKenzie \& Podsakoff, 2012).

En su formulación original, la escala de Barbara Larrivee consulta por la frecuencia en que se reportan distintas conductas asignadas a las dimensiones de prerreflexión, reflexión superficial, pedagógica y crítica. Para su elaboración, la autora estadounidense describió cada una de estas dimensiones a partir de una revisión de literatura. Con base en estas descripciones, un grupo de 40 investigadores sobre prácticas reflexivas elaboró ítems para cada dimensión. Luego de una primera revisión y pilotaje usando jueces, 76 de estos ítems fueron seleccionados. Estos ítems fueron clasificados por otro grupo de jueces en las cuatro dimensiones. Todos aquellos que no fueron clasificados de manera unívoca por la mayoría de los jueces fueron descartados. Por último, para reducir el número de ítems se retuvieron 53 entre aquellos que hubieran alcanzado el mayor consenso de los jueces en su clasificación.

Los ítems tienen una versión para ser usada por los formadores de profesores, con el fin de evaluar el nivel de reflexividad alcanzado por sus estudiantes de pedagogía, y otra versión para ser usada por los estudiantes de pedagogía con el fin de autoevaluar su nivel de reflexión. En el estudio que aquí se presenta se emplea la versión destinada a la autoevaluación, de modo que los datos obtenidos refieren a la percepción que los estudiantes de pedagogía tienen sobre la frecuencia de sus prácticas reflexivas.

Para efectos del trabajo que aquí se presenta, los ítems que miden el nivel de prerreflexión se descartaron porque no miden reflexividad, sino una etapa previa. De este modo, los tres niveles de reflexividad de Larrivee fueron medidos usando 39 de los ítems del instrumento original. Los ítems originales fueron traducidos al castellano y luego sometidos a una traducción en sentido inverso por un hablante nativo de inglés, con la finalidad de revisar la fidelidad de la traducción (los ítems originales y su traducción se pueden ver en el anexo).

La escala empleada en este estudio contiene 5 alternativas de respuesta: "muy frecuentemente" (5 puntos), "frecuentemente" (4 puntos), "rara vez" (3 puntos), "he tenido la oportunidad, pero nunca lo he hecho" (2 puntos) y "no he tenido la oportunidad" (1 punto).

Para validar la escala empleada, se realizó un análisis factorial exploratorio y confirmatorio. El análisis exploratorio mostró un Kaiser-MeyerOlkin $(\mathrm{KMO})=0,96$ y un test de Bartlett con $p<0,01$. Una explicación detallada del procedimiento y escala resultante se puede encontrar en Álvaro Salinas, Eugenio Chandía y Daniela Rojas (2017). 
Se analizaron varios modelos de 3 a 5 factores. En los primeros análisis se retuvieron 5 factores con autovalores superiores a 1. Luego de eliminar los ítems con cargas factoriales que no respetaban la formulación teórica original y de descartar el modelo con 4 factores que contenía uno con una varianza explicada de solo el $6 \%$, se llegó a un modelo de tres factores. Este modelo presentaba el mejor ajuste relativo $(\mathrm{CFI}=0,923 ; \mathrm{TLI}=0,915$; RMSEA $=0,057)$ y absoluto, con $x^{2}(249, n=489)=642,395, p<0,001$.

La escala finalmente empleada incluye 19 ítems, agrupados en tres factores. Uno de ellos pone su énfasis en "enseñanza", e incluye ítems que refieren la reflexión cuyo foco está en la flexibilidad en las prácticas docentes y en implementar soluciones a problemas con resultados de corto plazo, sin incorporar en la reflexión las consecuencias que la enseñanza podría tener en el aprendizaje. En este factor se pone atención en las estrategias, metodologías y soluciones a problemas, sin cuestionar el valor de estas para el aprendizaje de los alumnos. El segundo factor pone su énfasis en "enseñanza y aprendizaje", e incluye ítems con un foco de la reflexión docente puesto en el aprendizaje de los estudiantes, en la relación entre este aprendizaje y las prácticas del docente, y en cómo mejorar estas últimas. Este factor pone el foco en la relación entre las prácticas docentes y los resultados en el aprendizaje de los alumnos. Por último, un tercer factor pone su foco en "factores e implicancias de la enseñanza", e incluye ítems que refieren a las implicancias éticas, sociales, políticas de la enseñanza y a factores que inciden en la enseñanza, como las creencias y expectativas (tabla 1).

Tabla 1

Cargas factoriales en un modelo de tres factores para la escala de Barbara Larrivee (2008) modificada

\begin{tabular}{|c|c|c|}
\hline Factor & Ítem & Cargas \\
\hline \multirow[t]{3}{*}{ Enseñanza } & $\begin{array}{l}\text { Modifica las estrategias de enseñanza al cuestionar los supuestos implícitos sobre } \\
\text { la enseñanza y el aprendizaje. }\end{array}$ & 0,672 \\
\hline & $\begin{array}{l}\text { Realiza bastantes cambios en la metodología de enseñanza o evaluación para acoger las } \\
\text { diferencias individuales de los estudiantes. }\end{array}$ & 0,739 \\
\hline & Implementa soluciones a problemas que ponen su foco en resultados de corto plazo. & 0,592 \\
\hline \multirow{8}{*}{$\begin{array}{l}\text { Enseñanza y } \\
\text { aprendizaje }\end{array}$} & Se esfuerza por lograr el aprendizaje para todos los estudiantes. & 0,795 \\
\hline & Busca maneras de conectar los nuevos conceptos con el conocimiento previo de los estudiantes. & 0,766 \\
\hline & $\begin{array}{l}\text { Considera atentamente las conexiones entre las acciones del profesor y el aprendizaje } \\
\text { de los estudiantes. }\end{array}$ & 0,712 \\
\hline & Se hace autocríticas constructivas sobre su propia enseñanza. & 0,697 \\
\hline & Reconoce la complejidad de la dinámica del aula. & 0,673 \\
\hline & Considera el punto de vista de los estudiantes en la toma de decisiones. & 0,734 \\
\hline & Considera que la investigación puede aportar a la enseñanza. & 0,647 \\
\hline & Tiene compromiso con el aprendizaje permanente y la mejora de la práctica. & 0,756 \\
\hline \multirow{8}{*}{$\begin{array}{l}\text { Factores e } \\
\text { implicancias } \\
\text { de la } \\
\text { enseñanza }\end{array}$} & Cuando hay una incongruencia entre sus creencias y sus acciones, toma medidas para rectificar. & 0,515 \\
\hline & Identifica los supuestos y premisas que subyacen a su forma de ver la educación. & 0,629 \\
\hline & Considera las implicaciones éticas de las prácticas en el aula. & 0,702 \\
\hline & Favorece que sus estudiantes se hagan responsables de las implicancias sociales de sus acciones. & 0,627 \\
\hline & Reconoce las consecuencias sociales y políticas de su propia enseñanza. & 0,639 \\
\hline & $\begin{array}{l}\text { Cuestiona los supuestos sobre los estudiantes y las expectativas (positivas o negativas) } \\
\text { que hay sobre ellos. }\end{array}$ & 0,530 \\
\hline & Considera la práctica dentro de un contexto sociológico, cultural, histórico y político más amplio. & 0,540 \\
\hline & Analiza su propia forma de pensar. & 0,572 \\
\hline
\end{tabular}

Fuente: elaboración propia 
La varianza explicada por los tres factores alcanza el 50\% del modelo completo. El factor "enseñanza" explica el 19\% de la varianza, el factor "enseñanza y aprendizaje" explica el $16 \%$ y el factor "factores e implicancias de la enseñanza" explica el 15\%. El alfa de Cronbach para la escala completa alcanza 0,93. La subescala de reflexión sobre "enseñanza" incluye tres ítems y tiene un alfa de Cronbach de 0,7; la subescala de reflexión sobre "enseñanza y aprendizaje" incluye 8 ítems y alcanza un alfa de Cronbach de 0,9, y la subescala de reflexión sobre "factores e implicancias de la enseñanza" contiene 8 ítems y alcanza un alfa de Cronbach de 0,81.

Las variables independientes han sido:

1. La cantidad de prácticas profesionales en las cuales los estudiantes de pedagogía han participado durante sus estudios de pedagogía.
En la encuesta, los estudiantes debían indicar mediante una pregunta de respuesta abierta cuántas prácticas profesionales han tenido a lo largo de su formación profesional, en las cuales hubieran trabajado por al menos dos semanas en la escuela.

2. La frecuencia de actividades realizadas en el contexto de la supervisión. Los estudiantes de pedagogía fueron consultados sobre 16 actividades que podrían haber sido desarrolladas durante la supervisión (tabla 2). Los ítems que midieron estas actividades fueron redactados en forma de afirmaciones, y los estudiantes de pedagogía debían indicar la frecuencia con la cual ellos creían que se habían desarrollado esas actividades. La escala era de 4 puntos, donde 1 corresponde a "nunca" y 4 corresponde a "muy frecuentemente".

Tabla 2

Cargas factoriales en el análisis de componentes principales para las actividades

realizadas en el contexto de supervisión durante la práctica profesional

\begin{tabular}{|c|c|c|}
\hline Componente & Ítems & Cargas \\
\hline \multirow{7}{*}{$\begin{array}{l}\text { Relaciones, } \\
\text { aprendizaje de } \\
\text { los estudiantes }\end{array}$} & $\begin{array}{l}\text { Ha discutido sobre cómo manejar problemas de relación o convivencia con otros } \\
\text { profesores del establecimiento donde usted hace la práctica. }\end{array}$ & 0,78 \\
\hline & $\begin{array}{l}\text { Ha discutido sobre cómo manejar problemas de relación o convivencia con el profesor } \\
\text { del curso donde usted hace su práctica. }\end{array}$ & 0,735 \\
\hline & $\begin{array}{l}\text { Ha discutido sobre los errores comunes que cometen los alumnos al aprender } \\
\text { un cierto contenido. }\end{array}$ & 0,705 \\
\hline & Ha discutido sobre cómo trabajar con los padres y apoderados. & 0,685 \\
\hline & Ha discutido sobre cómo evaluar el aprendizaje de los estudiantes. & 0,634 \\
\hline & Ha discutido sobre las mejores estrategias para que los alumnos aprendan. & 0,62 \\
\hline & $\begin{array}{l}\text { Ha revisado y discutido la planificación de las clases que usted podría hacer } \\
\text { en el establecimiento educacional. }\end{array}$ & 0,496 \\
\hline \multirow{3}{*}{$\begin{array}{l}\text { Profesión docente, } \\
\text { valores y conductas } \\
\text { de los estudiantes }\end{array}$} & $\begin{array}{l}\text { Ha discutido sobre los problemas de la profesión docente o del sistema } \\
\text { educativo chileno. }\end{array}$ & 0,837 \\
\hline & Ha discutido sobre el rol de los profesores en la formación de valores de los alumnos. & 0,81 \\
\hline & $\begin{array}{l}\text { Ha discutido sobre problemas conductuales de los alumnos del curso donde } \\
\text { hace su práctica. }\end{array}$ & 0,64 \\
\hline \multirow[t]{3}{*}{$\begin{array}{l}\text { Compañeros } \\
\text { de práctica }\end{array}$} & $\begin{array}{l}\text { Ha observado clases realizadas por sus compañeros que están realizando la práctica } \\
\text { (directamente o por video). }\end{array}$ & 0,948 \\
\hline & $\begin{array}{l}\text { Ha sido observado por compañeros que están realizando la práctica mientras hace } \\
\text { clases (directamente o por video). }\end{array}$ & 0,942 \\
\hline & $\begin{array}{l}\text { Ha recibido retroalimentación significativa sobre su trabajo en la práctica, de parte } \\
\text { de compañeros que están realizando la práctica. }\end{array}$ & 0,328 \\
\hline \multirow[t]{3}{*}{ Supervisor } & Ha sido observado por su supervisor mientras hace clases (directamente o por video). & 0,752 \\
\hline & $\begin{array}{l}\text { Ha recibido retroalimentación significativa sobre su trabajo en la práctica de parte } \\
\text { del profesor supervisor, tutor o guía. }\end{array}$ & 0,801 \\
\hline & Ha analizado cómo usted podría hacer mejores clases. & 0,449 \\
\hline
\end{tabular}


Para explorar cómo estos 16 ítems pueden ser agrupados en dimensiones más amplias, se realizó un análisis de componentes principales. Para la escala completa, Kaiser-Meyer-Olkin (KMO) fue de 0,84 y el test de Bartlett alcanzó una $p<0,01$. Este análisis revela que los ítems pueden ser agrupados en cuatro componentes (tabla 2). El componente 1 incluye ítems relacionados con el aprendizaje de los alumnos y con las relaciones con los colegas y los padres. El segundo componente incluye ítems relacionados con la profesión docente, la formación de valores y el comportamiento de los alumnos. El tercer componente incluye ítems relacionados con los compañeros de práctica. Finalmente, el cuarto componente incluye ítems relacionados con la observación y retroalimentación que el supervisor hace del desempeño del estudiante durante la práctica.

Los cuatro componentes identificados explican en conjunto $60,3 \%$ de la varianza del modelo. El primero de ellos explica un 33\% de la varianza, el segundo un $12 \%$, el tercer componente explica un $8 \%$ y el cuarto explica un 7\%. El alfa de Cronbach para la escala completa alcanza 0,85. La subescala "relaciones y aprendizaje de los estudiantes" incluye 7 ítems y tiene un alfa de Cronbach de 0,85. La subescala "profesión docente, valores de los alumnos y conducta" incluye 3 ítems y alcanza un alfa de Cronbach de 0,79. La subescala "compañeros de práctica profesional" contiene 3 ítems y alcanza un alfa de Cronbach de 0,70. La subescala "supervisor" contiene 3 ítems y alcanza un alfa de Cronbach de 0,55.

\section{Análisis de datos}

Tres tipos de análisis de datos fueron realizados.

1. De tendencia central y dispersión para las tres subescalas de reflexividad y para el número de prácticas profesionales realizadas por los estudiantes de pedagogía. El puntaje de cada subescala de reflexividad fue calculado como el promedio simple del conjunto de ítems que la componen. Este puntaje fue el empleado para el análisis de regresión múltiple que se describe más abajo.

2. De correlación ( $r$ de Pearson) para analizar la fuerza de la relación entre las tres subescalas de reflexividad con el número de prácticas profesionales realizadas por los estudiantes de pedagogía y los 4 componentes que agrupan las actividades realizadas en el contexto de la supervisión de práctica.

3. De regresión múltiple, para analizar cuánto de las tres subescalas de reflexividad se explica por la cantidad de prácticas profesionales y por los 4 componentes que agrupan las actividades realizadas durante la supervisión; y cuáles de estas variables independientes contribuyen más a esta explicación. Considerando que estas variables independientes han sido descritas en investigaciones previas, se optó por un análisis de regresión con método "introducir". Para este análisis, el supuesto de ausencia de valores anómalos influyentes se cumple. Para las tres variables dependientes, el valor de la distancia de Cook $=0,002$. Lo mismo ocurre con el supuesto de homocedasticidad: los gráficos de dispersión entre los residuos estandarizados y los valores predichos estandarizados no muestran formas claras de túnel. Además, la multicolinealidad puede descartarse para "reflexión sobre la enseñanza" (Tolerancia oscila entre 0,59 y 0,92, y VIF [factor de varianza inflada] oscila entre 1,09 y 1,70), para "reflexión sobre la enseñanza y el aprendizaje" (Tolerancia oscila entre 0,59 y 0,92, y VIF oscila entre 1,09 y 1,71) y para "reflexión sobre los factores e implicancias de la enseñanza" (Tolerancia oscila entre 0,59 y 0,92 y VIF oscila entre 1,08 y 1,68). El supuesto de independencia también se cumple (para la variable "reflexión sobre la enseñanza", Durbin-Watson = 2,0; para la variable "reflexión sobre la enseñanza y aprendizaje", Durbin-Watson = 1,9; para la variable "reflexión sobre factores e implicancias de la enseñanza", Durbin-Watson $=2,0$ ). El supuesto de normalidad, sin embargo, no se cumple. El análisis de los residuos muestra pruebas Kolmogorov-Smirnov con resultados $p<0,05$ para todos los modelos. Sin embargo, es esperable una incidencia menor en el análisis de los datos dado el tamaño de la muestra empleada.

\section{Muestra}

La muestra fue seleccionada considerando las 5 regiones de Chile que concentran la mayor matrícula en programas de formación inicial de profesores. En estas regiones se ubica el $76 \%$ de los programas de formación de profesores del país y el $80 \%$ de la matrícula.

El estudio empleó un muestreo en dos etapas. Para seleccionar programas, se realizó un muestreo aleatorio simple entre los 261 incluidos en el repertorio de programas de formación inicial para profesores de educación básica y media ofrecidos en 2013 por las 52 instituciones de educación superior en Chile de las 5 regiones escogidas. Para seleccionar a los estudiantes de pedagogía, se hizo una selección aleatoria de cinco estudiantes de último año de formación pedagógica, usando para ello la lista de aquellos que estuvieron 
presentes en clases el día de aplicación. La encuesta era autoadministrada, y responderla tomaba alrededor de 20 minutos.

Empleando este procedimiento, se obtuvo información de 650 estudiantes de 134 programas de formación de profesores de enseñanza básica y media. Considerando varianza máxima ( $p=q=50 \%)$, el error muestral de los programas alcanza al $6 \%$.

La muestra obtenida está compuesta por un 58\% de mujeres y un $42 \%$ de hombres, con un promedio de edad de 24,6 años $(D . E .=4,5)$. El universo de referencia se compone de un 55\% de mujeres y de un $45 \%$ de hombres. Según estos datos, la muestra tiene un buen grado de ajuste con el universo, con una leve sobrerrepresentación de las mujeres y una subrepresentación de los hombres, lo que puede deberse a que la distribución de género en el universo considera a todos los alumnos matriculados en programas de formación de profesores, y no solo a los de último año, como ocurre en la muestra obtenida.

\section{Resultados}

Los datos muestran que de las tres subescalas que miden focos de las prácticas reflexivas, la reflexión sobre la enseñanza obtuvo la media más baja, con $M=3,53, D . E$. $=0,88$. La reflexión sobre los factores e implicancias de la enseñanza obtuvo $M=3,95, D . E .=0,66$. La reflexión sobre la enseñanza y el aprendizaje obtuvo el más alto promedio, con $M=4,35, D . E .=0,63$ (tabla 3). Los estudiantes encuestados señalaban que ellos realizaban de manera más bien infrecuente prácticas relacionadas con la reflexión con foco en la enseñanza, y de manera frecuente, prácticas reflexivas sobre la enseñanza y el aprendizaje y sobre los factores e implicancias de la enseñanza.

Tabla 3

Descriptivos para las subescalas de reflexión docente

\begin{tabular}{l|c|c|c|c|c}
\hline Subescala & n & M & D.E. & Mínimo & Máximo \\
\hline Reflexión sobre la enseñanza & 631 & 3,527 & 0,879 & 1,00 & 5,00 \\
\hline Reflexión sobre la enseñanza y aprendizaje & 629 & 4,346 & 0,630 & 1,00 & 5,00 \\
\hline Reflexión sobre los factores e implicancias de la enseñanza & 609 & 3,954 & 0,659 & 1,00 & 5,00 \\
\hline \hline
\end{tabular}

Fuente: elaboración propia

Factores asociados a la frecuencia de la práctica reflexiva

Las tres subescalas de reflexión docente están significativamente correlacionadas con el número de prácticas realizadas (tabla 4). La subescala que pone el foco en la reflexión sobre la relación entre la enseñanza y el aprendizaje obtuvo la mayor correlación de Pearson, con $r(628)=+0,302$, $p<0,01$, seguida de la reflexión sobre la enseñanza, con $r(630)=+0,299$, $p<0,01$, y finalmente la subescala sobre la reflexión sobre los factores e implicancias, con $r(609)=0,245, p<0,01$.

Las tres subescalas de práctica reflexiva tienen correlaciones de Pearson significativas con los cuatro componentes en los que se agrupan el conjunto de ítems que miden las actividades realizadas en la práctica profesional (tabla 4). Para la reflexión sobre la enseñanza, la mayor correlación se observa con el componente 4 (supervisor), con $r(570)=0,380$, $p<0,01$, mientras la menor correlación se observa con el componente 3 (compañeros de práctica), $r(561)=0,182, p<0,01$. Para la reflexión sobre la relación entre la enseñanza y el aprendizaje, la mayor correlación 
se obtiene con el componente 4 (supervisor), con $r(570)=+0,371, p<$ 0,01 , y la menor se obtiene con el componente 3 (compañeros de práctica), con $r(560)=+0,158, p<0,01$. Para la reflexión sobre factores e implicancias de la enseñanza, la correlación más alta es con el componente 4 (supervisor) con $r(552)=+0,370, p<0,01$, mientras la menor correlación se observa con el componente 3 (compañeros de práctica), con $r(540)=$ $+0,168, p<0,01$.

Tabla 4

Correlaciones de Pearson entre las tres subescalas de reflexión y variables independientes

\begin{tabular}{l|c|c|c|c|c|c}
\hline \multicolumn{2}{l|}{ Subescala } & $\begin{array}{l}\text { Número de } \\
\text { prácticas }\end{array}$ & $\begin{array}{l}\text { Relaciones, } \\
\text { aprendizaje }\end{array}$ & $\begin{array}{l}\text { Profesión } \\
\text { docente }\end{array}$ & Compañeros & Supervisor \\
\hline Enseñanza & $r$ & $0,299^{* *}$ & $0,296^{* *}$ & $0,239^{* *}$ & $0,182^{* *}$ & $0,380^{* *}$ \\
\hline Enseñanza y aprendizaje & $r$ & $0,302^{* *}$ & $0,308^{* *}$ & $0,336^{* *}$ & $0,158^{* *}$ & $0,371^{* *}$ \\
\hline $\begin{array}{l}\text { Factores e implicancias de } \\
\text { la enseñanza }\end{array}$ & $r$ & $0,245^{* *}$ & $0,352^{* *}$ & $0,351^{* *}$ & $0,168^{* *}$ & $0,370^{* *}$ \\
\hline \hline
\end{tabular}

${ }^{*} \mathrm{p}<0,05 ;{ }^{* *} \mathrm{p}<0,01$

Fuente: elaboración propia

Para analizar cuáles de las distintas variables independientes consideradas explican más los puntajes obtenidos en las tres subescalas, se realizó un análisis de regresión múltiple lineal. Para el análisis se consideró la cantidad de prácticas realizadas y los cuatro componentes que agrupan las actividades realizadas en el contexto de la supervisión.

Para la subescala de reflexión sobre la enseñanza, las variables incorporadas explican el $20 \%$ de la varianza, con $R^{2}=0,198, F(5,494)=$ $24,426, p<0,01$. La explicación que ofrece el modelo es estadísticamente significativa (tabla 5$)$.

Tres variables explican significativamente la variación de los puntajes de la subescala (tabla 5): componente 4 (supervisor), con Beta = $0,299, p<0,01$; componente 1 (relaciones, aprendizaje), con Beta $=0,17$, $p<0,01$, y el número de prácticas profesionales realizadas, con Beta $=$ $0,143, p<0,01$.

Tabla 5

Regresión múltiple para la subescala de reflexión sobre la enseñanza

\begin{tabular}{|c|c|c|c|c|c|}
\hline \multicolumn{2}{|c|}{ Modelo } & \multicolumn{2}{|c|}{$\begin{array}{l}\text { Coeficientes } \\
\text { no estandarizados }\end{array}$} & \multirow{3}{*}{$\begin{array}{l}\text { Coeficientes } \\
\text { estandarizados } \\
\text { Beta }\end{array}$} & \multirow{3}{*}{$\begin{array}{c}\mathbf{t} \\
7,241\end{array}$} \\
\hline & & \multirow{2}{*}{$\begin{array}{l}\text { B } \\
1,541\end{array}$} & \multirow{2}{*}{$\begin{array}{l}\text { Error estándar } \\
0,213 \\
\end{array}$} & & \\
\hline \multirow{6}{*}{1} & (Constante) & & & & \\
\hline & Componente 1 (relaciones, aprendizaje) & 0,219 & 0,068 & $0,170 * *$ & 3,234 \\
\hline & Componente 2 (profesión docente, rol) & $-0,026$ & 0,061 & $-0,021$ & $-0,420$ \\
\hline & Componente 3 (compañeros) & 0,018 & 0,046 & 0,017 & 0,394 \\
\hline & Componente 4 (supervisor) & 0,395 & 0,060 & $0,299 * *$ & 6,566 \\
\hline & Número de prácticas profesionales & 0,068 & 0,020 & $0,143^{* *}$ & 3,405 \\
\hline
\end{tabular}

$R^{2}=0,198, F(5,494)=24,426, p<0,01 .{ }^{*} p<0,05 ;{ }^{*} p<0,01$

Variable dependiente: subescala de reflexión sobre la enseñanza

Fuente: elaboración propia 
Para la reflexión sobre la enseñanza y el aprendizaje, las variables seleccionadas explican el $19 \%$ de la varianza, con $R^{2}=0,192, F(5,490)$ $=23,251, p<0,05$. El modelo explica la variación de los puntajes de la subescala de manera estadísticamente significativa (tabla 6).

Tal como muestra la tabla, las variables que más contribuyen a la explicación son: componente 4 (supervisor), con Beta $=0,242, p<0,05$, y el componente 2 (la profesión docente, el rol del profesor), con Beta = $0,159, p<0,05$.

Tabla 6

Regresión múltiple para la subescala de reflexión sobre la enseñanza y el aprendizaje

\begin{tabular}{l|l|l|l|l|l}
\hline \multicolumn{2}{l}{ Modelo } & \multicolumn{2}{l|}{$\begin{array}{l}\text { Coeficientes } \\
\text { no estandarizados }\end{array}$} & $\begin{array}{l}\text { Coeficientes } \\
\text { estandarizados }\end{array}$ & t \\
\cline { 2 - 6 } \multicolumn{2}{c|}{} & B & Error estándar & Beta & 23,162 \\
\hline \multirow{3}{*}{1} & (Constante) & 3,046 & 0,131 & & 1,980 \\
\cline { 2 - 6 } & Componente 1 (relaciones, aprendizaje) & 0,083 & 0,042 & $0,105^{*}$ & 3,084 \\
\cline { 2 - 6 } & Componente 2 (profesión docente, rol) & 0,116 & 0,038 &, $0159^{* *}$ & 0,046 \\
\cline { 2 - 6 } & Componente 3 (compañeros) & 0,001 & 0,028 & 0,002 & 5,317 \\
\cline { 2 - 6 } & Componente 4 (supervisor) & 0,195 & 0,037 & $0,242^{* *}$ & 2,789 \\
\cline { 2 - 6 } & Número de prácticas profesionales & 0,035 & 0,012 & $0,118^{* *}$ & \\
\hline
\end{tabular}

$R^{2}=0,192, F(5,490)=23,251, p<0,05 .{ }^{*} p<0,05 ;{ }^{* *} p<0,01$

Variable dependiente: subescala de reflexión sobre la enseñanza y el aprendizaje

Fuente: elaboración propia

Para la subescala de reflexión sobre los factores e implicancias de la enseñanza, las variables seleccionadas también explican el $19 \%$ de la varianza, con $R^{2}=0,193, F(5,478)=22,898, p<0,05$. La explicación que ofrece el modelo es estadísticamente significativa.

Las variables que más contribuyen a la explicación del modelo son: el componente 4 (supervisión), con Beta $=0,221, p<0,05$, y el componente 1 (relaciones, aprendizaje), con Beta $=0,171, p<0,05$ (tabla 7).

Tabla 7

Regresión múltiple para la subescala de factores e implicancias de la enseñanza

\begin{tabular}{l|l|l|l|l|c}
\hline \multicolumn{2}{l}{ Modelo } & \multicolumn{2}{l|}{$\begin{array}{l}\text { Coeficientes } \\
\text { no estandarizados }\end{array}$} & $\begin{array}{l}\text { Coeficientes } \\
\text { estandarizados }\end{array}$ & t \\
\cline { 2 - 6 } \multicolumn{2}{c|}{} & B & Error estándar & Beta & 15,459 \\
\hline \multirow{3}{*}{1} & (Constante) & 2,417 & 0,156 & & 3,208 \\
\cline { 2 - 6 } & Componente 1 (relaciones, aprendizaje) & 0,157 & 0,049 & $0,171^{* *}$ & 2,644 \\
\cline { 2 - 6 } & Componente 2 (profesión docente, rol) & 0,116 & 0,044 & $0,136^{* *}$ & 0,854 \\
\cline { 2 - 6 } & Componente 3 (compañeros) & 0,028 & 0,033 & 0,037 & 4,794 \\
\cline { 2 - 6 } & Componente 4 (supervisor) & 0,210 & 0,044 & $0,221^{* *}$ & 1,029 \\
\cline { 2 - 6 } & Número de prácticas profesionales & 0,015 & 0,015 & 0,044 & \\
\hline
\end{tabular}

$R^{2}=0,193, \mathrm{~F}(5,478)=22,898, \mathrm{p}<0,05 .{ }^{*} \mathrm{p}<0,05 ;{ }^{* *} \mathrm{p}<0,01$

Variable dependiente: subescala de reflexión sobre factores e implicancias de la enseñanza

Fuente: elaboración propia 


\section{Discusión y conclusiones}

Una de las preguntas de investigación que ha guiado este trabajo ha sido: ¿qué actividades específicas realizadas en el marco de la práctica profesional contribuyen más a desarrollar las prácticas reflexivas en estudiantes de último año de pedagogía en Chile? Los datos muestran que las actividades relacionadas con el supervisor de la práctica no solo están correlacionadas de manera estadísticamente significativa con las tres subescalas de prácticas reflexivas, sino que además son las más importantes para explicarlas. De este modo, los datos obtenidos ofrecen evidencia que permite retener las hipótesis número 3, que señala que mientras más observación, retroalimentación y análisis sobre el desempeño de los estudiantes de pedagogía ofrezca el supervisor, mayor es la frecuencia de prácticas reflexivas de los estudiantes de pedagogía de último año. Además, los datos permiten retener la hipótesis número 4, que señala que estas actividades son las que mayor peso tienen para explicar las prácticas reflexivas de estos estudiantes, en comparación con las otras variables consideradas en el estudio.

Las actividades relacionadas con "relaciones y aprendizaje" muestran correlaciones positivas y significativas estadísticamente con las tres subescalas de prácticas reflexivas, y contribuyen también, aunque menos que el componente relacionado con el supervisor, a explicar la reflexión sobre "enseñanza" y reflexión sobre "factores e implicancias de la enseñanza". Entre las actividades relacionadas con "relaciones y aprendizaje" está discutir durante la supervisión sobre la relación con otros profesores, sobre cómo trabajar con los padres, sobre los errores de los alumnos, sobre la evaluación y sobre la planificación de clases. De este modo, la hipótesis 7 puede retenerse considerando que, a mayor frecuencia de actividades de este tipo en el contexto de la supervisión, mayor frecuencia de prácticas reflexivas.

Las actividades relacionadas con la discusión sobre la "profesión docente y el rol de los profesores", también tienen correlaciones estadísticamente significativas con las tres subescalas de prácticas reflexivas. Sin embargo, contribuyen solo a explicar la reflexión sobre "enseñanza y aprendizaje". Estas actividades incluyen: discutir sobre los desafíos de la profesión docente en Chile, sobre el rol de los profesores en la formación de valores de los alumnos y sobre problemas conductuales de los alumnos. Con ello, la hipótesis 6 puede ser retenida, considerando que a mayor discusión sobre la profesión y el rol docente, mayor frecuencia de prácticas reflexivas.

Las actividades relacionadas con "compañeros de práctica" también están relacionadas de manera significativa con las tres subescalas de prácticas re- flexivas, de manera tal que es posible retener la hipótesis 5. Sin embargo, estas actividades no contribuyen a explicar de manera significativa ninguna de las tres subescalas, a pesar de la bibliografía que destaca la contribución de la discusión y acompañamiento entre pares en contextos de práctica profesional (Harford \& MacRuairc, 2008).

Los datos encontrados indican que la reflexión docente se desarrolla especialmente en un contexto de interacción con el supervisor, y que este es clave en la medida en que movilice la experiencia del estudiante de pedagogía a través de la retroalimentación y del examen de la práctica. Los compañeros parecen contribuir, pero no tan fuertemente, tal vez porque la retroalimentación que puedan proveer carece del mismo potencial de movilizar la reflexión que logra el supervisor. Estos datos van en la misma línea desarrollada por Andrea Gelfuso y Danielle V. Dennis (2014), que destacan la importancia de la interacción con "otros conocedores" para desarrollar la reflexión durante la experiencia de práctica profesional. Con todo, es necesaria más investigación para comprender cuál es la contribución específica y bajo qué condiciones (si acaso), el trabajo entre compañeros puede contribuir más a las prácticas reflexivas.

La contribución que hacen el conjunto de estas actividades realizadas en el contexto de la supervisión indica que su riqueza y diversidad son claves para desarrollar en los estudiantes de pedagogía la reflexión sobre la enseñanza y el aprendizaje, situados en el contexto específico donde estos ocurren. Los datos encontrados permiten señalar que para que los estudiantes de pedagogía desarrollen esos distintos focos, se requiere ofrecerles oportunidades de aprendizaje diversas, entre las cuales la discusión y retroalimentación del supervisor son claves, pero también lo son las oportunidades para discutir sobre la profesión docente, sobre las relaciones con los colegas y los padres, tal como lo señalan Alisa J. Bates, Laurie Ramírez y Dina Drits (2009), Georgea M. Sparks-Langer, Joanne M. Simmons, Marvin Pasch, Amy Colton y Alane Starko (1990).

La cantidad de prácticas profesionales también muestra correlaciones estadísticamente significativas con las tres subescalas de prácticas reflexivas, de modo que la hipótesis 2 puede ser retenida. Esta hipótesis señala que a mayor cantidad de prácticas profesionales, mayor frecuencia de prácticas reflexivas. La cantidad de prácticas profesionales contribuye a explicar la reflexión sobre "enseñanza" y la reflexión sobre "enseñanza y aprendizaje", aunque esta contribución es menor que la que se aprecia para las actividades de supervisión. Tal como indican Simon Collin, Thierry Karsenti y Vassilis Komis (2012), Andrea Gelfuso y Danielle V. Dennis (2014), la práctica profesional ofrece 
un vínculo con contextos reales de desempeño, y esto tendría una incidencia importante en el desarrollo de dos de los tres focos de las prácticas reflexivas. Con todo, los datos muestran además que más importante que la cantidad de prácticas es cómo las actividades realizadas en el contexto de la supervisión que la acompaña son aprovechadas, lo que apunta en la misma línea que otros trabajos (Bates, Ramírez \& Drits, 2009; Gelfuso \& Dennis, 2014).

Otra pregunta que ha guiado este trabajo ha sido si, en el contexto chileno, los estudiantes de pedagogía reflexionan con mayor frecuencia sobre la enseñanza y con menos frecuencia sobre el aprendizaje y sobre las mediaciones sociales y políticas de su trabajo. Mucha investigación realizada hasta ahora ha mostrado que los estudiantes de pedagogía tienden más bien a describir su propia enseñanza, con poco uso de distinciones propias del campo pedagógico y sin considerar dimensiones como el aprendizaje de sus alumnos ni el contexto de su labor (Collin, Karsenti \& Komis, 2012; Mansvelder-Longayroux, Beijaard \& Verloop, 2007). Este planteamiento ha sido recogido en la hipótesis 1 . Sin embargo, este estudio ha mostrado que los estudiantes de pedagogía reflexionan más frecuentemente sobre la relación entre "enseñanza y aprendizaje", seguido de la reflexión sobre "Ios factores e implicancias de la enseñanza" y de la reflexión sobre la "enseñanza". Esto significa que, según los datos obtenidos, esta hipótesis no tendría sustento.

Estos resultados son distintos a los que reporta la literatura. De esto se pueden hacer dos posibles interpretaciones. La primera de ellas es que el contexto de este estudio podría explicar unos resultados distintos a los que se tienden a encontrar en los países donde se ha realizado más investigación sobre práctica reflexiva. En este sentido, los estudiantes de pedagogía de último año en el contexto chileno, a diferencia de sus pares en otros contextos nacionales, tendrían un mayor foco en la relación entre la enseñanza y el aprendizaje y en los factores e implicancias de la enseñanza. Para profundizar en esto se requiere de investigación que pueda comparar distintos contextos nacionales, analizando por ejemplo diferencias entre las culturas profesionales o los sistemas de formación de profesores. La segunda es que el tipo de instrumento empleado incide en apreciaciones más positivas respecto de la importancia de reflexionar sobre el aprendizaje de los estudiantes y el contexto de trabajo. Como se ha dicho, la escala empleada recoge la percepción que los propios estudiantes de pedagogía tienen sobre la frecuencia de sus prácticas reflexivas. En este sentido, los resultados reflejarían más bien lo que los estudiantes piensan que es la práctica deseable, y por ello se aprecia un reporte en el cual la frecuencia de la reflexión sobre la enseñanza y el aprendizaje es mayor que el encontrado por otros estudios. Se requiere más investigación para avanzar en la comprensión de las relaciones entre las percepciones de los propios estudiantes y sus prácticas reflexivas efectivas.

Este artículo siguió una perspectiva cuantitativa para estudiar las prácticas reflexivas. Los datos y las conclusiones obtenidas aquí complementan, enriquecen y permiten una comprensión más amplia de las prácticas reflexivas, tema que ha sido estudiado usando fundamentalmente perspectivas cualitativas. La opción de desarrollar más investigación cuantitativa que emplee muestras grandes, estudie distintos factores y mida con precisión los fenómenos parece ser una perspectiva prometedora. Para hacer esto, más y mejores instrumentos cuantitativos debieran ser desarrollados. La escala modificada de Larrivee empleada aquí es una buena opción, pero para usar con mayor confianza el instrumento empleado aquí se requieren varias cosas. 
En primer lugar, se requiere analizar con mayor profundidad los tres factores que resultaron del análisis factorial, para comprender la composición de cada factor, las relaciones entre ellos y por qué algunas actividades realizadas en el contexto de la práctica se relacionan más con algunos de estos factores y menos con otros. El factor que pone el foco de la reflexión en la enseñanza se concentra en la actividad realizada por el propio estudiante. Este factor recoge y precisa lo que Barbara Larrivee en su propuesta inicial de instrumento empleado en este trabajo, llamaba "nivel de reflexión superficial", en el cual se pone atención a los métodos y estrategias para alcanzar determinados fines (la enseñanza), pero sin preguntarse por el valor de esos fines (el aprendizaje). El factor que pone el foco en la relación entre la enseñanza y el aprendizaje incorpora una mayor complejidad en la medida en que implica una relación que incorpora un elemento que le da sentido a la práctica educativa: el aprendizaje. Este factor recoge y precisa lo que Larrivee llamaba "nivel de reflexión pedagógica", según el cual los profesores aplican criterios profesionales para definir una práctica de calidad (aquella que logra el aprendizaje). Ambos factores son abordados por Deborah Moore-Russo y Jillian N. Wilsey (2014) cuando describe el foco que los estudiantes de pedagogía ponen en su propia enseñanza (su instrucción, sus atributos como docentes) o en el aprendizaje de los alumnos. El tercer factor que pone el foco sobre los factores e implicancias de la enseñanza, recoge y precisa lo que Larrivee llama "nivel de reflexión crítica".

En segundo lugar, se requiere profundizar en la validación del instrumento empleado aquí, comparando, por ejemplo, sus resultados con los obtenidos con otros instrumentos o bien usando grupos contrastados. El instrumento empleado recoge datos de autorreporte. La principal limitación de este tipo de datos es el potencial sesgo producido por la deseabilidad social al momento de responder al cuestionario. Sin embargo, más que descartar el autorreporte en estudios sobre prácticas reflexivas, lo que hay que hacer es profundizar sobre cuán válida y confiable es esta clase de medida. Los trabajos sobre el sesgo en instrumentos de recolección de datos tienden a coincidir en que este varía según el constructo y que los instrumentos cuantitativos ofrecen ventajas que pueden complementar la investigación cualitativa (Podsakoff, MacKenzie \& Podsakoff, 2012).

En tercer lugar, este trabajo estudió la contribución que hacen la práctica profesional y las actividades que se realizan en el contexto de la supervisión a la práctica reflexiva. El análisis aquí privilegió una mirada general y de una escala mayor a la que usualmente se observa en la investigación cualitativa sobre la práctica reflexiva. El procedimiento de selección de casos y el buen ajuste que se observa entre la muestra y el universo de estudio permiten extrapolar con cierta confianza las conclusiones obtenidas a los estudiantes de último año de pedagogía de las regiones seleccionadas en Chile. Con todo, la perspectiva empleada sacrificó el estudio detallado y en profundidad sobre cómo los distintos tipos de actividad inciden en la reflexión docente, qué y cómo se discuten los distintos temas en el contexto de la supervisión, cuáles son los estilos de supervisión y cómo se desarrolla la interacción entre el supervisor, el estudiante de pedagogía y sus pares, etc. El uso complementario de investigación cualitativa y cuantitativa, con las fortalezas y debilidades de cada una, puede ayudar a una mejor comprensión del fenómeno complejo y sobre el que poco se sabe en el contexto latinoamericano. 


\section{Agradecimientos}

Este trabajo ha sido financiado por el Fondo Nacional de Desarrollo Científico y Tecnológico, Fondecyt (1120834).

\section{Sobre los autores}

Álvaro Luis Salinas-Espinosa es académico, Facultad de Educación, Pontificia Universidad Católica de Chile, Chile.

Tamara Rozas-Assael es investigadora en la Facultad de Educación, Pontificia Universidad Católica de Chile y en CIAE, Universidad de Chile, Chile.

Pablo Cisternas-Alarcón es ayudante de investigación en la Facultad de Educación, Pontificia Universidad Católica de Chile, Chile.

Carlos González-Ugalde es académico, Facultad de Educación, Pontificia Universidad Católica de Chile, Chile.

\section{Referencias}

Bates, A. J.; Ramírez, L. \& Drits, D. (2009). Connecting University Supervision and Critical Reflection: Mentoring and Modeling. The Teacher Educator, 44 (2), 90-112. doi: 10.1080/08878730902751993

Beauchamp, C. (2014). Reflection in Teacher Education: Issues Emerging from a Review of Current Literature. Reflective Practice, 16 (1), 123141. doi: 10.1080/14623943.2014.982525

Collin, S.; Karsenti, T. \& Komis, V. (2012). Reflective Practice in Initial Teacher Training: Critiques and Perspectives. Reflective Practice, 14 (1), 104-117. doi: 10.1080/14623943.2012.732935. Disponible en: http:// www.karsenti.ca/archives/CollinKarsentiKomis2013.pdf

Darling-Hammond, L. \& Lieberman, A. (2012). Teacher Education around the World. What Can We Learn from International Practice? En L. Darling-Hammond \& A. Lieberman (eds.). Teacher Education Around the World: Changing Policies and Practices, 151-169. New York: Routledge.

Gelfuso, A. \& Dennis, D. V. (2014). Getting Reflection Off the Page: The Challenges of Developing Support Structures for Pre-Service Teacher Reflection. Teaching and Teacher Education, 38, 1-11. doi: http://dx.doi. org/10.1016/j.tate.2013.10.012. Disponible en: https://s3.amazonaws. com/arena-attachments/272964/edd6eab9dd18ee89bc6b8b 913fb9818d.pdf

Harford, J. \& MacRuairc, G. (2008). Engaging Student Teachers in Meaningful Reflective Practice. Teaching and Teacher Education, 24 (7), 1884-1892. Disponible en: http://eprints.teachingandlearning. ie/2428/1/Harford\%20and\%20MacRuairc\%202008.pdf

Ingvarson, L.; Schwille, J.; Tatto, M. T.; Rowley, G.; Peck, R. \& Senk, S. L. (2013). An Analysis of Teacher Education Context, Structure, and Quality-Assurance Arrangements in TEDS-M Countries. New Zealand: International Association for the Evaluation of Educational Achievement, IEA. Disponible en: https://www.iea.nl/fileadmin/user_upload/ Publications/Electronic_versions/TEDS-M_Findings.pdf

Jaeger, E. (2013). Teacher Reflection: Supports, Barriers, and Results. Issues in Teacher Education, 22 (1), 89-104. Disponible en: https://files.eric. ed.gov/fulltext/EJ1014037.pdf 
Jones, M. \& Ryan, J. (2014). Learning in the Practicum: Engaging PreService Teachers in Reflective Practice in the Online Space. AsiaPacific Journal of Teacher Education, 42 (2), 132-146. doi: 10.1080/ 1359866x.2014.892058

Kember, D.; Leung, D. Y. P.; Jones, A.; Loke, A. Y.; McKay, J., Sinclair, K.; Tse, H.; Webb, C.; Yuet Wong, F. K.; Wong, M. \& Yeung, E. (2000). Development of a Questionnaire to Measure the Level of Reflective Thinking. Assessment \& Evaluation in Higher Education, 25 (4), 381 395. doi: 10.1080/026029300449272

Larrivee, B. (2008). Development of a Tool to Assess Teachers' Level of Reflective Practice. Reflective Practice: International and Multidisciplinary Perspectives, 9 (3), 341-360. https://doi.org/10.1080/14623940 802207451

Leijen, Ä.; Valtna, K.; Leijen, D. A. J. \& Pedaste, M. (2012). How to Determine the Quality of Students' Reflections? Studies in Higher Education, 37 (2), 203-217. doi: 10.1080/03075079.2010.504814

Mansvelder-Longayroux, D. D.; Beijaard, D. \& Verloop, N. (2007). The Portfolio as a Tool for Stimulating Reflection by Student Teachers. Teaching and Teacher Education, 23 (1), 47-62. doi: http://dx.doi. org/10.1016/j.tate.2006.04.033. Disponible en: http://depts.wash ington.edu/comgrnd/ccli/papers/Mansvelder-Longayroux_etal_Port folioAsToolStimulatingReflectionByStudentTeachers-jy.pdf

Meckes, L. (2014). Estándares y formación docente inicial. En UNESCO/ OREALC (ed.). Temas críticos para formular nuevas políticas docentes en América Latina y el Caribe: el debate actual, 53-110. Santiago de Chile: UNESCO/OREALC.

Moore-Russo, D. A. \& Wilsey, J. N. (2014). Delving into the Meaning of Productive Reflection: A Study of Future Teachers' Reflections on Representations of Teaching. Teaching and Teacher Education, 37 (1), 76-90. DOI: 10.1016/j.tate.2013.10.002

Podsakoff, P.; MacKenzie, S. \& Podsakoff, N. (2012). Sources of Method Bias in Social Science Research and Recommendations on How to Control it. Annual Review of Psychology, 63, 539-569. Disponible en: https://www.annualreviews.org/doi/abs/10.1146/annurevpsych-120710-100452

Salinas, A.; Chandía, E. \& Rojas, D. (2017). Validación de un instrumento cuantitativo para medir la práctica reflexiva de docentes en formación. Revista Estudios Pedagógicos, XLIII (1), 289-309. Disponible en: https://scielo.conicyt.cl/pdf/estped/v43n1/art17.pdf

Schön, D. A. (1983). The Reflective Practitioner: How Professionals Think In Action. London, New York: Basic Books.

Sobral, D. T. (2001). Medical Students' Reflection in Learning in Relation to Approaches to Study and Academic Achievement. Medical Teacher, 23 (5), 508-513. DOI: 10.1080/01421590120042973

Solís, M. C.; Núñez, C.; Contreras, I.; Rittershaussen, S.; Montecinos, C. \& Walker, H. (2011). Condiciones de la formación práctica de los futuros profesores. Estudios Pedagógicos (Valdivia), 37, 127-147. Disponible en: https://scielo.conicyt.cl/pdf/estped/v37n1/art07.pdf

Sparks-Langer, G. M.; Simmons, J. M.; Pasch, M.; Colton, A. \& Starko, A. (1990). Reflective Pedagogical Thinking: How Can We Promote It and Measure It? Journal of Teacher Education, 41 (5), 23-32. doi: $10.1177 / 002248719004100504$ 
Anexo 1

Ítems originales de la Escala de Autoevaluación del Desarrollo de la Práctica Reflexiva de Barbara Larrivee y su traducción al castellano, indicando la subescala a la cual pertenece cada ítem

\begin{tabular}{|c|c|c|c|}
\hline Ítems originales & $\begin{array}{l}\text { Nivel de } \\
\text { reflexión } \\
\text { original }\end{array}$ & Ítems traducidos & $\begin{array}{l}\text { Subescala } \\
\text { final }\end{array}$ \\
\hline $\begin{array}{l}\text { Adjusts methods and strategies based on } \\
\text { students' relative performance }\end{array}$ & Pedagógica & $\begin{array}{l}\text { Ajusta las metodologías y las estrategias según el } \\
\text { rendimiento de los estudiantes }\end{array}$ & \\
\hline $\begin{array}{l}\text { Provides limited accommodations for } \\
\text { students' different learning styles }\end{array}$ & Superficial & $\begin{array}{l}\text { Realiza pocos cambios en la metodología de } \\
\text { enseñanza o evaluación, según los distintos } \\
\text { estilos de aprendizaje de los alumnos }\end{array}$ & \\
\hline $\begin{array}{l}\text { Acknowledges what student brings } \\
\text { to the learning process }\end{array}$ & Pedagógica & $\begin{array}{l}\text { Considera en el proceso de aprendizaje las } \\
\text { experiencias y conocimientos previos que el } \\
\text { estudiante trae consigo }\end{array}$ & \\
\hline Calls commonly-held beliefs into question & Crítica & $\begin{array}{l}\text { Pone en tela de juicio creencias sobre educación } \\
\text { comúnmente aceptadas por los profesores }\end{array}$ & \\
\hline $\begin{array}{l}\text { Addresses issues of equity and social } \\
\text { justice that arise in and outside of } \\
\text { the classroom }\end{array}$ & Crítica & $\begin{array}{l}\text { Contribuye a solucionar problemas de equidad y } \\
\text { justicia social que afectan a los estudiantes }\end{array}$ & \\
\hline $\begin{array}{l}\text { Supports beliefs only with evidence } \\
\text { from experience }\end{array}$ & Superficial & $\begin{array}{l}\text { Considera que basta con su experiencia para } \\
\text { fundamentar sus creencias }\end{array}$ & \\
\hline $\begin{array}{l}\text { Searches for patterns, relationships and } \\
\text { connections to deepen understanding }\end{array}$ & Pedagógica & $\begin{array}{l}\text { Busca patrones, relaciones y conexiones para } \\
\text { profundizar la comprensión de la situación de } \\
\text { aprendizaje }\end{array}$ & \\
\hline $\begin{array}{l}\text { Modifies teaching strategies without } \\
\text { challenging underlying assumptions about } \\
\text { teaching and learning }\end{array}$ & Superficial & $\begin{array}{l}\text { Modifica las estrategias de enseñanza } \\
\text { cuestionando los supuestos implícitos sobre la } \\
\text { enseñanza y el aprendizaje }\end{array}$ & Enseñanza \\
\hline $\begin{array}{l}\text { Acknowledges that teaching practices } \\
\text { and policies can either contribute to, or } \\
\text { hinder, the realization of a more just and } \\
\text { humane society }\end{array}$ & Crítica & $\begin{array}{l}\text { Tiene en cuenta que la enseñanza puede } \\
\text { contribuir —o bien impedir_a la realización de } \\
\text { una sociedad más justa y humana }\end{array}$ & \\
\hline $\begin{array}{l}\text { Fails to connect specific methods to } \\
\text { underlying theory }\end{array}$ & Superficial & $\begin{array}{l}\text { Tiene dificultades para conectar métodos de } \\
\text { enseñanza con teorías implícitas }\end{array}$ & \\
\hline Strives to enhance learning for all students & Pedagógica & $\begin{array}{l}\text { Se esfuerza por lograr el aprendizaje para todos } \\
\text { los estudiantes }\end{array}$ & $\begin{array}{l}\text { Enseñanza y } \\
\text { aprendizaje }\end{array}$ \\
\hline $\begin{array}{l}\text { Has genuine curiosity about the } \\
\text { effectiveness of teaching practices, } \\
\text { leading to experimentation and risk taking }\end{array}$ & Pedagógica & $\begin{array}{l}\text { Se esfuerza por experimentar y asumir riesgos } \\
\text { para mejorar la efectividad de las prácticas de } \\
\text { enseñanza }\end{array}$ & \\
\hline $\begin{array}{l}\text { Limits analysis of teaching practices } \\
\text { to technical questions about } \\
\text { teaching techniques }\end{array}$ & Superficial & $\begin{array}{l}\text { Limita el análisis de las prácticas de enseñanza } \\
\text { solo a cuestiones técnicas }\end{array}$ & \\
\hline $\begin{array}{l}\text { Seeks ways to connect new concepts to } \\
\text { students' prior knowledge }\end{array}$ & Pedagógica & $\begin{array}{l}\text { Busca maneras de conectar los nuevos conceptos } \\
\text { con el conocimiento previo de los estudiantes }\end{array}$ & $\begin{array}{l}\text { Enseñanza y } \\
\text { aprendizaje }\end{array}$ \\
\hline $\begin{array}{l}\text { Analyzes the impact of task structures, } \\
\text { such as cooperative learning groups, } \\
\text { partner, peer or other groupings, on } \\
\text { students' learning }\end{array}$ & Pedagógica & $\begin{array}{l}\text { Analiza el impacto que tienen las distintas } \\
\text { metodologías de trabajo en clases (tales como } \\
\text { grupos de aprendizaje cooperativo, trabajo entre } \\
\text { pares u otras) en el aprendizaje de los alumnos }\end{array}$ & \\
\hline $\begin{array}{l}\text { Suspends judgments to consider } \\
\text { all options }\end{array}$ & Crítica & $\begin{array}{l}\text { Deja de lado algunos juicios para considerar } \\
\text { todas las opciones posibles }\end{array}$ & \\
\hline $\begin{array}{l}\text { Provides some differentiated instruction to } \\
\text { address students' individual differences }\end{array}$ & Superficial & $\begin{array}{l}\text { Realiza bastantes cambios en la metodología } \\
\text { de enseñanza o evaluación para acoger las } \\
\text { diferencias individuales de los estudiantes }\end{array}$ & Enseñanza \\
\hline $\begin{array}{l}\text { Questions the utility of specific } \\
\text { teaching practices but not general } \\
\text { policies or practices }\end{array}$ & Superficial & $\begin{array}{l}\text { Se centra en los aspectos específicos de una } \\
\text { clase (metodologías, recursos, actividades, etc.) } \\
\text { sin mirar el conjunto de la unidad u objetivos de } \\
\text { aprendizajes más a largo plazo }\end{array}$ & \\
\hline $\begin{array}{l}\text { Adjusts teaching practices only to } \\
\text { current situation without developing } \\
\text { a long-term plan }\end{array}$ & Superficial & $\begin{array}{l}\text { Ajusta las prácticas de enseñanza solo a las } \\
\text { situaciones actuales, sin desarrollar un plan a } \\
\text { largo plazo }\end{array}$ & \\
\hline
\end{tabular}




\begin{tabular}{|c|c|c|c|}
\hline ítems originales & \begin{tabular}{|l|} 
Nivel de \\
reflexión \\
original
\end{tabular} & Ítems traducidos & $\begin{array}{l}\text { Subescala } \\
\text { final }\end{array}$ \\
\hline $\begin{array}{l}\text { Is aware of incongruence between beliefs } \\
\text { and actions and takes action to rectify } \\
\text { challenges assumptions about students } \\
\text { and expectations for students }\end{array}$ & Crítica & $\begin{array}{l}\text { Cuando hay una incongruencia entre sus } \\
\text { creencias y sus acciones, toma medidas } \\
\text { para rectificar }\end{array}$ & $\begin{array}{l}\text { Factores e } \\
\text { implicancias de } \\
\text { la enseñanza }\end{array}$ \\
\hline $\begin{array}{l}\text { Analyzes relationship between teaching } \\
\text { practices and student learning }\end{array}$ & Pedagógica & $\begin{array}{l}\text { Considera atentamente las conexiones entre las } \\
\text { acciones del profesor y el aprendizaje de } \\
\text { los estudiantes }\end{array}$ & $\begin{array}{l}\text { Enseñanza y } \\
\text { aprendizaje }\end{array}$ \\
\hline $\begin{array}{l}\text { Engages in constructive criticism of one's } \\
\text { own teaching }\end{array}$ & Pedagógica & $\begin{array}{l}\text { Se hace autocríticas constructivas sobre su } \\
\text { propia enseñanza }\end{array}$ & $\begin{array}{l}\text { Enseñanza y } \\
\text { aprendizaje }\end{array}$ \\
\hline $\begin{array}{l}\text { Recognizes the complexity of } \\
\text { classroom dynamics }\end{array}$ & Pedagógica & Reconoce la complejidad de la dinámica del aula & $\begin{array}{l}\text { Enseñanza y } \\
\text { aprendizaje }\end{array}$ \\
\hline $\begin{array}{l}\text { Considers students' perspectives in } \\
\text { decision making }\end{array}$ & Pedagógica & $\begin{array}{l}\text { Considera el punto de vista de los estudiantes } \\
\text { en la toma de decisiones }\end{array}$ & $\begin{array}{l}\text { Enseñanza y } \\
\text { aprendizaje }\end{array}$ \\
\hline $\begin{array}{l}\text { Is an active inquirer, both critiquing } \\
\text { current conclusions and generating new } \\
\text { hypotheses calls commonly-held beliefs } \\
\text { into question }\end{array}$ & Crítica & $\begin{array}{l}\text { Es un investigador activo en la sala de clases, } \\
\text { que cuestiona conclusiones actuales y genera } \\
\text { nuevas hipótesis }\end{array}$ & \\
\hline $\begin{array}{l}\text { Recognizes assumptions and premises } \\
\text { underlying beliefs }\end{array}$ & Crítica & $\begin{array}{l}\text { Identifica los supuestos y premisas que subyacen } \\
\text { a su forma de ver la educación }\end{array}$ & $\begin{array}{l}\text { Factores e } \\
\text { implicancias de } \\
\text { la enseñanza }\end{array}$ \\
\hline $\begin{array}{l}\text { Considers the ethical ramifications of } \\
\text { classroom policies and practices }\end{array}$ & Crítica & $\begin{array}{l}\text { Considera las implicaciones éticas de las prácticas } \\
\text { en el aula }\end{array}$ & $\begin{array}{l}\text { Factores e } \\
\text { implicancias de } \\
\text { la enseñanza }\end{array}$ \\
\hline $\begin{array}{l}\text { Encourages socially responsible actions in } \\
\text { their students }\end{array}$ & Crítica & $\begin{array}{l}\text { Favorece que sus estudiantes se hagan } \\
\text { responsables de las implicancias sociales de } \\
\text { sus acciones }\end{array}$ & $\begin{array}{l}\text { Factores e } \\
\text { implicancias de } \\
\text { la enseñanza }\end{array}$ \\
\hline $\begin{array}{l}\text { Sees teaching practices as remaining open } \\
\text { to further investigation }\end{array}$ & Pedagógica & $\begin{array}{l}\text { Considera que la investigación puede aportar a } \\
\text { la enseñanza }\end{array}$ & $\begin{array}{l}\text { Enseñanza y } \\
\text { aprendizaje }\end{array}$ \\
\hline $\begin{array}{l}\text { Makes adjustments based on } \\
\text { past experience }\end{array}$ & Superficial & $\begin{array}{l}\text { Hace ajustes a su docencia basados en su } \\
\text { experiencia pasada (exitosa o no exitosa) }\end{array}$ & \\
\hline $\begin{array}{l}\text { Acknowledges the social and political } \\
\text { consequences of one's teaching }\end{array}$ & Crítica & $\begin{array}{l}\text { Reconoce las consecuencias sociales y políticas } \\
\text { de su propia enseñanza }\end{array}$ & $\begin{array}{l}\text { Factores e } \\
\text { implicancias de } \\
\text { la enseñanza }\end{array}$ \\
\hline $\begin{array}{l}\text { Reacts to student responses differentially } \\
\text { but fails to recognize patterns }\end{array}$ & Superficial & $\begin{array}{l}\text { No logra reconocer patrones comunes en las } \\
\text { diferentes acciones de sus estudiantes }\end{array}$ & \\
\hline $\begin{array}{l}\text { Challenges assumptions about students } \\
\text { and expectations for students }\end{array}$ & Crítica & $\begin{array}{l}\text { Cuestiona los supuestos sobre los estudiantes y } \\
\text { las expectativas (positivas o negativas) que hay } \\
\text { sobre ellos }\end{array}$ & $\begin{array}{l}\text { Factores e } \\
\text { implicancias de } \\
\text { la enseñanza }\end{array}$ \\
\hline $\begin{array}{l}\text { Has commitment to continuous learning } \\
\text { and improved practice }\end{array}$ & Pedagógica & $\begin{array}{l}\text { Tiene compromiso con el aprendizaje } \\
\text { permanente y la mejora de la práctica }\end{array}$ & $\begin{array}{l}\text { Enseñanza y } \\
\text { aprendizaje }\end{array}$ \\
\hline $\begin{array}{l}\text { Implements solutions to problems that } \\
\text { focus only on short-term results }\end{array}$ & Superficial & $\begin{array}{l}\text { Implementa soluciones a problemas que ponen } \\
\text { su foco en resultados de corto plazo }\end{array}$ & Enseñanza \\
\hline $\begin{array}{l}\text { Views practice within the broader } \\
\text { sociological, cultural, historical, and } \\
\text { political contexts }\end{array}$ & Crítica & $\begin{array}{l}\text { Considera la práctica dentro de un contexto } \\
\text { sociológico, cultural, histórico y político } \\
\text { más amplio }\end{array}$ & $\begin{array}{l}\text { Factores e } \\
\text { implicancias de } \\
\text { la enseñanza }\end{array}$ \\
\hline Observes self in the process of thinking & Crítica & Analiza su propia forma de pensar & $\begin{array}{l}\text { Factores e } \\
\text { implicancias de } \\
\text { la enseñanza }\end{array}$ \\
\hline $\begin{array}{l}\text { Challenges status quo norms and } \\
\text { practices, especially with respect to power } \\
\text { and control }\end{array}$ & Crítica & $\begin{array}{l}\text { Cuestiona la situación actual de normas y } \\
\text { prácticas educativas respecto al poder y el } \\
\text { control en la sala de clases }\end{array}$ & \\
\hline $\begin{array}{l}\text { Identifies alternative ways of representing } \\
\text { ideas and concepts to students }\end{array}$ & Pedagógica & $\begin{array}{l}\text { Conoce formas alternativas de representar ideas } \\
\text { y conceptos para enseñar a los estudiantes }\end{array}$ & \\
\hline
\end{tabular}

Fuente de los ítems originales: Larrivee, 2008. Traducción propia 